\title{
Amplitude-Phase Decorrelation: A Method for Reducing Intensity Noise in Semiconductor Lasers
}

\author{
Michael A. Newkirk and Kerry J. Vahala, Member, IEEE
}

\begin{abstract}
By the method of amplitude-phase decorrelation, the funAbstract-By the method of amplitude-phase decorrelation, the funduced over a wide bandwidth by the ratio $1 /\left(1+\alpha^{2}\right)$, where $\alpha$ is the linewidth enhancement factor. The method uses a dispersive element to convert phase noise into intensity noise. We recently demonstrated this technique by reducing intensity noise from a DFB laser as much as $7 \mathrm{~dB}$ below its intrinsic level [1], [2]. In this paper, we extend these results by characterizing the frequency dependence of the noise reduction. Optimum reduction is achieved in the flat region of the spectrum and diminishes at higher frequencies approaching the relaxation resonance. The correlation properties of the fluctuations are also investigated. The cross-spectral density of the fluctuations shows they are decorrelated when noise is maximally reduced. Parallels between this technique and "detuned loading" [17] will also be established.
\end{abstract}

\section{INTRODUCTION}

T HE linewidth enhancement factor, or $\alpha$ parameter, has been studied extensively in research on semiconductor lasers This parameter governs many of the important features of semiconductor laser dynamics, among them enhanced linewidth [3] and frequency chirping under direct modulation (dynamic line broadening) [4], [5]. In a semiconductor laser, the resonant refractive index and the gain are functions of carrier concentration. The amplitude and phase of the lasing field are therefore strongly coupled through the carrier density in the active medium. The $\alpha$ parameter is simply a measure of the coupling strength. However, the coupling is not symmetric. Field amplitude fluctuations are coupled into phase fluctuations, but not the reverse. With regard to laser noise, this enhances the phase noise (i.e., linewidth), but the intensity noise level is unaffected. Nevertheless, because of the inherent correlation between the fluctuations, an image of the intensity noise lies in the phase noise. We propose that this image can be recovered from the phase fluctuations and used to reduce the intensity noise far below its intrinsic level. When the intensity noise is maximally reduced, the amplitude and phase fluctuations become decorrelated.

We present a theoretical discussion of two methods to achieve intensity noise reduction. The first approach employs a passive element with a frequency-dependent transmission which is external to the laser cavity. Light from the free-running laser is passively processed by the transmission function, and a large reduction in intensity noise results. Recently, we demonstrated

Manuscript received June 6, 1990; revised August 24, 1990. This work was supported in part by the National Science Foundation. The work of K. J. Vahala was supported by a Richard P. Feynman Hughes Fellowship.

The authors are with the Department of Applied Physics, California Institute of Technology, Pasadena, CA 91125.

IEEE Log Number 9040997. this approach by reducing the intensity noise level from a DFB laser by $7 \mathrm{~dB}[1],[2]$. The theoretical basis of the idea was outlined, and the effect of a power independent component of laser linewidth on the noise reduction was also considered. In this paper, we review the previous results for completeness, and add to them by investigating the spectrum of noise reduction, as well as the correlation properties of the transformed field fluctuations. We also describe a second approach, whereby a frequency-dependent loss is placed inside the laser cavity. The effects due to such a dispersive intracavity loss have been considered previously in the context of detuned loading [17], but application to intensity noise reduction was not developed. Both methods can potentially reduce the fundamental intensity noise floor by the factor $1 /\left(1+\alpha^{2}\right)$, well over an order of magnitude for typical values of $\alpha$. Finally, results of the experiment which verify the first approach are discussed.

\section{Semiconductor Laser NoISE}

In this section we outline the main features of intrinsic noise in semiconductor lasers which will be important for later discussion of noise reduction. A semiclassical description of the field fluctuations incorporating Langevin noise sources will be used to derive the relevant noise spectra and their dependence on laser power. At the center of this discussion is the $\alpha$ parameter, which determines the coupling between amplitude and phase fluctuations in the field. Ultimately, we will show that this coupling can be used to reduce the intensity noise far below its intrinsic level

The electric field from a single-mode laser can be described as follows:

$$
E(t)=\sqrt{p_{0}+p(t)} e^{i[\omega L t+\varphi(t)]}
$$

where $p_{o}$ is the average photon density in the lasing mode, $p(t)$ is the fluctuating part of the photon density, $\omega_{L}$ is the $\mathrm{CW}$ oscillation frequency, and $\varphi(t)$ is the fluctuating phase deviation. When $p(t) / p_{0} \ll 1$ the field can be represented in terms of a dimensionless fluctuating amplitude

$$
E(t)=\sqrt{p_{o}}(1+\rho(t)) e^{i\left[\omega_{L} t+\varphi(t)\right]}
$$

where $\rho(t)=p(t) / 2 p_{o}$. As depicted in the intuitive model of Henry [6], the fluctuations arise from spontaneous emission which randomly perturbs the amplitude and phase of the field phasor. Since a semiconductor laser operates as a detuned os cillator (i.e., the gain peak lies at a frequency different from the zero dispersion point of the resonant refractive index) the amplitude fluctuations cause enhanced phase fluctuations by modulating the carrier-density-dependent refractive index of the gain 
medium. This phase noise enhancement is measured by the $\alpha$ parameter, given in terms of the real and imaginary parts of the complex susceptibility function as

$$
\alpha=\frac{d \chi_{R}(n) / d n}{d \chi_{l}(n) / d n}
$$

where $n$ is the carrier density. As defined, $\alpha$ is a negative number. For DFB semiconductor lasers, $\alpha$ may range from -2 to -7 depending on device design [7].

Each of the quantities $\rho(t), \varphi(t)$, and $n(t)$, the deviation of carrier density from the steady-state value, evolves according to the small-signal rate equations

$$
\begin{aligned}
& \dot{\rho}(t)=G^{\prime} n / 2+\Delta_{R} \\
& \dot{\varphi}(t)=-\alpha G^{\prime} n / 2+\Delta_{t} \\
& \dot{n}(t)=-n / \tau_{R}-2 p_{o} G \rho
\end{aligned}
$$

where $G$ is the optical gain, $G^{\prime}$ is the derivative of gain with respect to carrier density, $\tau_{R}$ is the relaxation oscillation damping time, and $\Delta_{R}$ and $\Delta_{l}$ are real and imaginary parts of the Langevin force accounting for spontaneous emission into the lasing mode [8]. We omit a noise term in the carrier density equation because it has a negligible effect on the dynamics of $\rho(t)$ and $\varphi(t)$ at low frequencies where gain clamping is operative [9]. The Langevin forcing terms are assumed to come from a zero-mean Gaussian probability distribution and have the correlation relations [10]

$$
\begin{aligned}
\left\langle\Delta_{R}(t+\tau) \Delta_{R}(t)\right\rangle & =\frac{S}{2 P} \delta(\tau) \\
\left\langle\Delta_{I}(t+\tau) \Delta_{I}(t)\right\rangle & =\frac{S}{2 P} \delta(\tau) \\
\left\langle\Delta_{R}(t+\tau) \Delta_{I}(t)\right\rangle & =0
\end{aligned}
$$

where $S$ is the spontaneous emission rate into the lasing mode, $P$ is the average photon number in the mode, and $\delta(\tau)$ is the Dirac delta function. The angle brackets denote ensemble averaging. By Fourier transforming the above rate equations with respect to frequency $\Omega$, the dynamics of $n$ can be absorbed into equations for $\rho$ and $\dot{\varphi}$, the instantaneous frequency deviation, to obtain the fluctuation spectra

$$
\begin{gathered}
\rho(\Omega)=\frac{\frac{1}{\tau_{R}}+i \Omega}{\left(\omega_{R}^{2}-\Omega^{2}\right)+\frac{i \Omega}{\tau_{R}}} \tilde{\Delta}_{R} \\
\dot{\varphi}(\Omega)=\tilde{\Delta}_{l}+\frac{\alpha \omega_{R}^{2}}{\left(\omega_{R}^{2}-\Omega^{2}\right)+\frac{i \Omega}{\tau_{R}}} \tilde{\Delta}_{R} .
\end{gathered}
$$

Here, $G G^{\prime} p_{o}$ has been replaced by $\omega_{R}^{2}$, the relaxation oscillation frequency. By Parseval's theorem, the transformed Langevin terms $\tilde{\Delta}_{R}$ and $\tilde{\Delta}_{l}$ are delta correlated in frequency with the same normalizations as given in (5).

Equations (6a) and (6b) will provide the foundation for the following discussion of noise reduction. In these equations, the role of $\alpha$ is seen more clearly as providing the coupling of amplitude fluctuations into instantaneous frequency fluctuations. It should be emphasized that this is a one-way coupling, from $\rho$ to $\dot{\varphi}$. The amplitude fluctuation spectrum is thus not affected by gain spectrum detuning and the $\alpha$ parameter.
One consequence of a nonzero $\alpha$, however, is enhancement of the fundamental linewidth over its Schawlow-Townes value. Using the low frequency limit of (6b), one finds that the power spectrum of the field is a Lorentzian with a linewidth [6]

$$
\Delta \omega=\Delta \omega_{\mathrm{ST}}\left(1+\alpha^{2}\right)
$$

where

$$
\Delta \omega_{\mathrm{ST}}=\frac{S}{2 P}
$$

is the modified Schawlow-Townes linewidth. In the ideal case, linewidth is inversely proportional to laser power. However, at high power, it is often observed that the linewidth reaches a constant value and thereafter remains independent of power. To account for this extra component of linewidth, a power-independent term will be added to (7), so that

$$
\Delta \omega=\Delta \omega_{\mathrm{ST}}\left(1+\alpha^{2}\right)+\Delta \omega_{\rho} .
$$

The origin of this extraneous linewidth is not known, but several potential mechanisms have been discussed in the literature. These include carrier number fluctuations [11], thermal fluctuations [12], and spatial hole burning [13]. In any case, the existence of a power-independent source of linewidth can be incorporated into the instantaneous frequency fluctuation spectrum by adding a phenomenological noise source $\tilde{\Delta}_{o}$, such that $\Delta \omega_{o}$ is the spectral density of $\tilde{\Delta}_{o}$. Equation (6b) then becomes

$$
\dot{\varphi}(\Omega)=\tilde{\Delta}_{l}+\frac{\alpha \omega_{R}^{2}}{\left(\omega_{R}^{2}-\Omega^{2}\right)+\frac{i \Omega}{\tau_{R}}} \tilde{\Delta}_{R}+\tilde{\Delta}_{\omega} .
$$

It is assumed that $\tilde{\Delta}_{o}$ is uncorrelated with the other Langevin source arising from spontaneous emission. The presence of this term will later illustrate how an independent source of linewidth influences our ability to reduce intensity noise.

For a directly detected field, the relative intensity noise (RIN) is defined as the ratio of the mean square power per unit bandwidth of the fluctuating photocurrent to the average photocurrent power. By this definition, the RIN spectrum is directly proportional to the spectral density of the amplitude fluctuations, given by

$$
W_{\rho \rho}(\Omega)=\left\langle\rho^{*}(\Omega) \rho(\Omega)\right\rangle=\frac{\frac{1}{\tau_{R}^{2}}+\Omega^{2}}{\left(\omega_{R}^{2}-\Omega^{2}\right)^{2}+\frac{\Omega^{2}}{\tau_{R}^{2}}} \Delta \omega_{\mathrm{S} \mathrm{S}} .
$$

Since the proportionality constant is of no consequence when direct comparisons of RIN are made, hereafter we will define relative intensity noise as

$$
\mathrm{RIN} \equiv W_{\rho \rho}(\Omega) .
$$

The way in which the RIN varies as a function of laser power can be divided into two regimes. For the moment, only consider fluctuation frequencies $\Omega \ll 1 / \tau_{R}, \omega_{R}$, so that the intensity noise has a flat spectrum given by

$$
\mathrm{RIN}=\frac{S}{2 P \tau_{R}^{2} \omega_{R}^{4}}
$$

At low power, $\tau_{R}$ is essentially constant while $\omega_{R}^{2}$ is always linearly proportional to $P$. This characterizes the excess noise regime, where the RIN falls as $1 / P^{3}$. In the high-power limit, $\tau_{R} \omega_{R}^{2}$ saturates to a constant value, and so the RIN falls as $1 / P$, 
which is defined as the shot noise regime. In practice, both types of behavior can be easily observed by directly detecting the intensity noise with a photodiode followed by a high-gain amplifier.

For the remainder of this paper, we will assume that the laser is operating in the excess noise regime when we discuss the method of amplitude-phase decorrelation for reducing intensity noise in the field. The laser noise is then sufficiently far above the level of vacuum field noise that a semiclassical treatment can be applied to all aspects of the problem. This is not to say that the noise reduction method we propose is invalid for a laser operating in the shot noise regime, however. In that case, we must address the question of reducing noise below the shot noise level, which lies in the realm of squeezed light generation [14]. A quantum operator description of the laser field, the vacuum fluctuations, and the noise measurement process is then required. This treatment will be presented elsewhere [15].

To conclude this section, we note that for a given operating point, (11) represents the intensity noise floor of the laser field. As previously stated, this intrinsic noise level is not a function of $\alpha$. Even so, we demonstrate in the next two sections that the amplitude-phase coupling determined by $\alpha$ can be used to reduce intensity noise below its fundamental floor.

\section{Intensity Noise Reduction with a Passive External Transmission FUnCtion}

In this section we discuss a scheme to reduce semiconductor laser intensity noise which exploits the fundamental amplitudephase coupling in the field fluctuations [1], [2]. The technique relies on the observation that although the intensity noise level is independent of $\alpha$, the enhanced instantaneous frequency fluctuations measured by $\alpha$ contain an image of the intensity noise. It is this image resulting from the correlation between $\rho(\Omega)$ and $\dot{\varphi}(\Omega)$ which can be recovered to reduce the intensity noise below its intrinsic level. In what follows, we show that an external, passive element with a frequency dependent transmission (see Fig. 1) can accomplish this task by transforming the field in the desired way. We emphasize that no feedback to the laser source is involved in this scheme. The fluctuating field is passively processed after leaving the laser cavity. For an ideal laser source with no extraneous component of linewidth, the intensity noise can potentially be reduced by the amount $1 /\left(1+\alpha^{2}\right)$, the inverse of the linewidth broadening term. This reduction is independent of laser power, and is achieved by altering the correlation between the fluctuating quantities. We quantify the effect of the transmission function on the field's correlation properties by calculating the cross-spectral density of the fluctuations. For the ideal laser mentioned above, maximum intensity noise reduction will coincide with decorrelation of amplitude and instantaneous frequency fluctuations. Even in the case of a laser with an extraneous linewidth component, large reductions in intensity noise exceeding an order of magnitude are possible. The dependence of noise reduction on output power will then be discussed.

\section{A. Transformation of Field Fluctuations}

Before analyzing the noise reduction method quantitatively, we can form an intuitive picture of how a frequency-dependent transmission function affects the field fluctuations. Suppose that we send the radiation through such a function $T(\omega)$. The fluctuations in instantaneous frequency can be viewed as the lasing frequency jittering about the lasing linecenter $\omega_{L}$. Assuming that

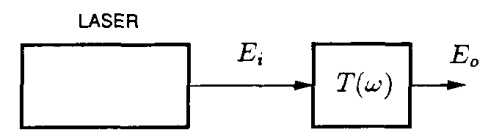

Fig. 1. Free-running laser and passive, external transmission function $T(\omega)$. The intrinsic intensity noise in the input light can be reduced when $T(\omega)$ has the right slope.

$T(\omega)$ has nonzero slope in the vicinity of $\omega_{L}$, the frequency jitter is converted into jitter in the transmitted amplitude. Since these new amplitude fluctuations are correlated with the intrinsic amplitude fluctuations in the field, they can be made to cancel each other when they are superposed. Thus, the intensity noise can potentially be reduced by choosing a proper transmission function.

It is straightforward to evaluate the effect of the transmission function on the fluctuations [2]. Assume that $T(\omega)$ is slowly varying over the range of fluctuation frequencies we are interested in. It will be seen that this is an excellent assumption when $T(\omega)$ is realized by a Michelson interferometer. A Taylor expansion of $T$ about the lasing line center is then possible, yielding

$$
T\left(\omega_{L}+\Omega\right) \approx T\left(\omega_{L}\right)+\Omega T^{\prime}\left(\omega_{L}\right)
$$

where $T^{\prime}$ is the derivative of the transmission function with respect to frequency. In terms of a Fourier component of the input and output fields, we then have

$$
\begin{aligned}
\tilde{A}_{o}(\Omega) & =T\left(\omega_{L}+\Omega\right) \tilde{A}_{i}(\Omega) \\
& =T\left(\omega_{L}\right) \tilde{A}_{i}(\Omega)+\Omega T^{\prime}\left(\omega_{L}\right) \tilde{A}_{i}(\Omega)
\end{aligned}
$$

where the slowly varying amplitude is defined as

$$
A(t)=[1+\rho(t)] e^{i \varphi(t)}
$$

so that $E(t)=A(t) \sqrt{p_{o}} \exp \left(i \omega_{L} t\right)$ [see (2)]. By inspection, $(15)$ is the Fourier transform of the equation

$$
A_{o}(t)=T\left(\omega_{L}\right) A_{i}(t)-i T^{\prime}\left(\omega_{L}\right) \dot{A}_{i}(t)
$$

which can be used to derive the effect of $T(\omega)$ on the amplitude fluctuations in the output field, with the result,

$$
\rho_{o}(t)=T \rho_{i}(t)+T^{\prime}\left[\dot{\varphi}_{i}(t)-i \dot{\rho}_{i}(t)\right] .
$$

Similarly, the derivative with respect to time of (17) in conjunction with (18) gives

$$
\dot{\varphi}_{o}(t)=\dot{\varphi}_{i}(t) .
$$

Products of small-signal quantities have been neglected in writing the above relations. Transforming back, we find

$$
\begin{aligned}
& \rho_{o}(\Omega)=\left(T_{R}+\Omega T_{R}^{\prime}\right) \rho_{i}(\Omega)+T_{R}^{\prime} \dot{\varphi}_{i}(t) \\
& \dot{\varphi}_{o}(\Omega)=\dot{\varphi}_{i}(\Omega)
\end{aligned}
$$

where the subscript $R$ denotes the real part of $T$.

Equation (20a) shows that $\rho_{v}(\Omega)$ is the sum of two parts depending on both the amplitude fluctuation and instantaneous frequency fluctuation of the input field. The inherent correlation between $\rho_{i}$ and $\dot{\varphi}_{i}$ will enable intensity noise reduction at the output by controlling $T$ and $T^{\prime}$. The instantaneous frequency fluctuation of the output field, on the other hand, is not affected by the transmission function, as (20b) shows. Thus, we usually will not make a distinction between $\dot{\varphi}_{i}$ and $\dot{\varphi}_{o}$.

To account for the fact that $T(\omega)$ attenuates the mean field in addition to transforming the fluctuations, we normalize $\rho_{o}(\Omega)$ 
by $T_{R}$ and so obtain

$$
\rho_{o}(\Omega)=(1+\Omega \xi) \rho_{i}(\Omega)+\xi \dot{\varphi}_{i}(\Omega)
$$

where $\xi \equiv T_{R}^{\prime} / T_{R}$ and will hereafter be referred to as the "slope" of $T$. By making this definition, the RIN spectra of the input and output are simply

$$
\begin{aligned}
& \operatorname{RIN}_{i}=W_{\rho_{i} \rho_{i}}(\Omega) \\
& \operatorname{RIN}_{o}=W_{\rho_{i} \rho_{i}}(\Omega)
\end{aligned}
$$

and we see that $\mathrm{RIN}_{o} \rightarrow \mathrm{RIN}_{i}$ as $\xi \rightarrow 0$, that is, in the limit of zero transmission function slope.

In reality, the factor $\Omega \xi$ appearing in the transformation equation (21) is usually much smaller than one and can be neglected for the range of frequencies and slopes that will be encountered. In particular, the results of Section $\mathrm{V}$ will show that to achieve noise reduction, $\xi$ will typically be $0.03 \mathrm{GHz}^{-1}$ or less, so $\Omega \xi$ $\ll 1$ for all frequencies of interest. With this observation, (21) becomes

$$
\rho_{o}(\Omega)=\rho_{i}(\Omega)+\xi \dot{\varphi}_{i}(\Omega)
$$

which is the final transformation equation.

\section{B. Spectral Density of Transformed Fluctuations}

If we assume for now that we have an ideal laser source, that is, one without an extraneous linewidth component, then the spectral density of the output amplitude fluctuations may be computed using (6a) and (6b).

$$
\operatorname{RIN}_{o}=\left[\xi^{2}+\frac{\left(1 / \tau_{R}+\alpha \xi \omega_{R}^{2}\right)^{2}+\Omega^{2}}{\left(\omega_{R}^{2}-\Omega^{2}\right)^{2}+\frac{\Omega^{2}}{\tau_{R}^{2}}}\right] \Delta \omega_{\mathrm{ST}} .
$$

This expression simplifies if we restrict our attention to frequencies $\Omega \ll 1 / \tau_{R}, \omega_{R}$, where the RIN spectrum is flat. In this case

$$
\operatorname{RIN}_{o}=\left[\xi^{2}+\frac{\left(1 / \tau_{R}+\alpha \xi \omega_{R}^{2}\right)^{2}}{\omega_{R}^{4}}\right] \Delta \omega_{\mathrm{ST}}
$$

and using

$$
\mathrm{RIN}_{i}=\frac{\Delta \omega_{\mathrm{ST}}}{\tau_{R}^{2} \omega_{R}^{4}}
$$

the RIN of the input and output fields may be easily related.

$$
\operatorname{RIN}_{o}=\left[\left(\tau_{R} \omega_{R}^{2} \xi\right)^{2}+\left(1+\alpha \tau_{R} \omega_{R}^{2} \xi\right)^{2}\right] \operatorname{RIN}_{i} .
$$

By minimizing this function with respect to $\xi$, the optimum slope is found to be

$$
\xi_{\mathrm{opt}}=\frac{-1}{\tau_{R} \omega_{R}^{2}} \frac{\alpha}{1+\alpha^{2}}
$$

which gives the maximum noise reduction

$$
\mathrm{RIN}_{o}=\frac{1}{1+\alpha^{2}} \mathrm{RIN}_{i}
$$

For a typical $\alpha=-5$, the intensity noise floor can be reduced below its intrinsic level by a factor of 26 . This lower limit on noise reduction is governed by the factor $\left(1+\alpha^{2}\right)$, the same factor which enhances the fundamental linewidth of the laser over its Schawlow-Townes value. As the slope is changed from its optimum value, different amounts of noise reduction (or en-

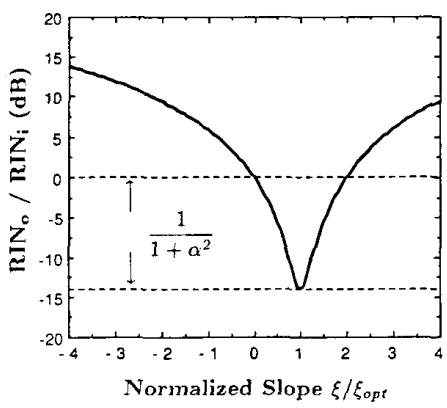

Fig. 2. Relative level of output intensity noise versus normalized slope For the optimum slope (28), noise is reduced below its intrinsic level by $1 /\left(1+\alpha^{2}\right)$, where $\alpha=-5$ is assumed. This figure characterizes noise reduction in the flat part of the intensity noise spectrum.

hancement) will occur in accordance with (27). This is shown in Fig. 2 where the output noise is seen to vary quadratically with slope about the minimum. According to (28), the optimum slope is a function of $\tau_{R} \omega_{R}^{2}$ and generally will vary with laser bias point. Even so, the maximum reduction (29) remains independent of laser power.

The complete RIN spectrum of the output field is given by (24). For a given value of $\xi$, the amount of noise reduction is a function of frequency. Fig. 3 shows the RIN spectrum calculated from (24) for several values of transmission slope. Typical values for $\tau_{R}\left(2.6 \times 10^{-10} \mathrm{~s} / \mathrm{rad}\right)$ and $\omega_{R}\left(9.4 \times 10^{9} \mathrm{rad} / \mathrm{s}\right)$ characteristic of a DFB laser at low bias are taken from the literature [16]. An $\alpha$ of -5 is also used. The heavy curve is the intrinsic intensity noise level of the input field, corresponding to $\xi=0$. When $\xi$ is at the optimum value given by (28), the low-frequency noise is reduced by $1 /\left(1+\alpha^{2}\right)$ in accordance with (29), but the amount of reduction diminishes at higher frequencies. If the slope is too large, $3 \xi_{\text {opt }}$ for this example, then the low-frequency noise is enhanced over its intrinsic value, in agreement with (27) and Fig. 2. For the range of slopes in the figure, the noise level at the resonance is not noticeably affected. We also notice that the high-frequency noise beyond the resonance is enhanced even when the low-frequency noise is reduced. This is not surprising because at high frequencies the phase noise is dominated by its independent component $\left[\tilde{\Delta}_{I}\right.$ term in (6b)] which is being converted to an additive component of intensity noise.

The optimum slope which minimizes the output intensity noise at frequency $\Omega$ is

$$
\xi_{\text {opt }}(\Omega)=\frac{-1}{\tau_{R} \omega_{R}^{2}} \frac{\alpha D(\Omega)}{1+\alpha^{2} D(\Omega)}
$$

where

$$
D(\Omega)=\frac{\omega_{R}^{4}}{\left(\omega_{R}^{2}-\Omega^{2}\right)^{2}+\frac{\Omega^{2}}{\tau_{R}^{2}}} .
$$

The variation in optimum slope is shown in Fig. 4, where $\xi_{\text {opt }}(\Omega)$ is plotted normalized by the optimum low-frequency slope $\xi_{\text {opt }}$ from (28). Noise reduction at higher frequencies therefore requires a fairly constant slope as $\Omega$ approaches $\omega_{R}$. Beyond the resonance the optimum slope tends to zero, which indicates that noise reduction is becoming impossible at these high frequencies.

A plot of the maximum achievable noise reduction versus fre- 


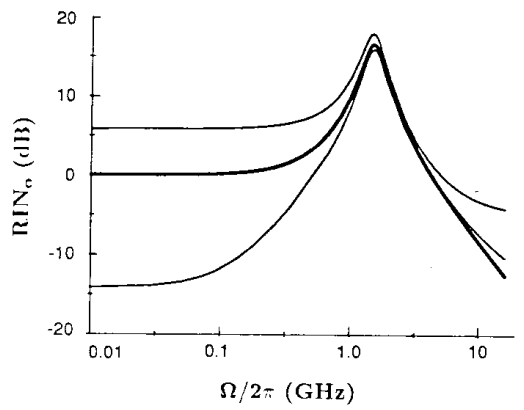

Fig. 3. Output intensity noise spectrum for different slopes calculated from (24). The heavy curve is the intrinsic noise level $(\xi=0)$. Spectra are measured relative to intrinsic noise level at low frequency. Noise is reduced in flat region by $1 /\left(1+\alpha^{2}\right)$ when $\xi=\xi_{\text {upt }}$, or enhanced $(\xi=$ $\left.3 \xi_{\text {opt }}\right)$ as slope is varied. High-frequency noise is enhanced for all slopes.

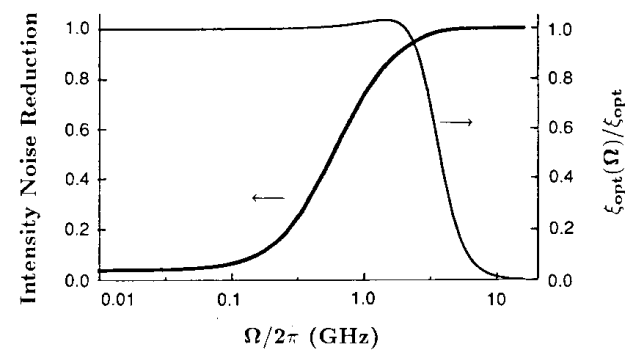

Fig. 4. Left-hand side: maximum intensity noise reduction as a function of frequency. Noise is reduced by $1 /\left(1+\alpha^{2}\right)$ where the noise spectrum is flat. Beyond the resonance, noise cannot be reduced below the intrinsic level. Right-hand side: normalized optimum slope to achieve intensity noise reduction versus frequency. $\xi_{\text {upl }}(\Omega)$ tends to zero at high frequency where noise reduction is not possible.

quency also appears in Fig. 4. This function is obtained by eval-

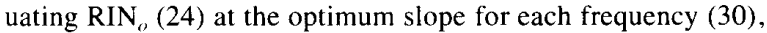
and is the envelope of minimum noise levels in the progression of noise spectra in Fig. 3, normalized by the intrinsic noise spectrum. This shows how the noise can be reduced by $1 /(1$ $+\alpha^{2}$ ) at low frequency, the magnitude of reduction decreases as the resonance frequency is approached, and no reduction is possible at frequencies beyond the resonance.

\section{Correlation Properties}

To further illustrate the effect of the transmission function $T(\omega)$ on the field fluctuations, it is useful to compute the symmetric cross-spectral density of $\rho$ and $\dot{\varphi}$, defined as

$$
W_{\rho \dot{\varphi}}(\Omega)=\frac{1}{2}\left[\left\langle\rho(\Omega) \dot{\varphi}^{*}(\Omega)\right\rangle+\left\langle\rho^{*}(\Omega) \dot{\varphi}(\Omega)\right\rangle\right] .
$$

The cross-spectral density of the input field is then

$$
W_{\rho i \varphi}(\Omega)=\frac{\frac{\alpha \omega_{R}^{2}}{\tau_{R}}}{\left(\omega_{R}^{2}-\Omega^{2}\right)^{2}+\frac{\Omega^{2}}{\tau_{R}^{2}}} \Delta \omega_{\mathrm{ST}} .
$$

This function gives the degree of correlation between the Fourier components of the field's intrinsic amplitude and instantaneous frequency fluctuations. For the input field, a logarithmic plot of the magnitude of (33) in Fig. 5 shows that there is always correlation except in the high-frequency limit, which fits with the intuitive model of [6]. Since the fluctuations are cou-

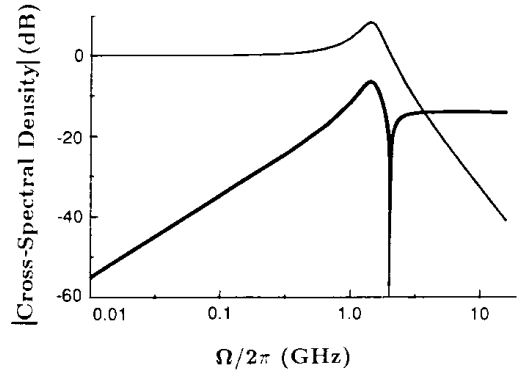

Fig. 5. Magnitude of cross-spectral density of $\rho(\Omega)$ and $\dot{\varphi}(\Omega)$ of the input field normalized by low-frequency value, and of the output field (heavy curve) for $\xi=\xi_{\text {op }}$, showing decorrelation of the fluctuations at low frequency. The function changes sign after the resonance, appearing as a sharp feature of this logarithmic plot.

pled through perturbations to the carrier density, there cannot be any significant correlation at timescales much shorter than the characteristic response time $\tau_{R}$. As expected, the above function is directly proportional to $\alpha$. To be precise, the fact that $\alpha$ is negative means that $\rho_{i}$ and $\dot{\varphi}$ are anticorrelated.

After passing through the transmission function, the amplitude fluctuation is transformed according to (23), and the crossspectral density of the output field becomes

$$
W_{\rho, \varphi}(\Omega, \xi)=\left[\xi+\frac{\alpha^{2} \omega_{R}^{4} \xi+\frac{\alpha \omega_{R}^{2}}{\tau_{R}}}{\left(\omega_{R}^{2}-\Omega^{2}\right)^{2}+\frac{\Omega^{2}}{\tau_{R}^{2}}}\right] \Delta \omega_{\mathrm{ST}} .
$$

The correlation is now a function of $\xi$, the transmission function slope. Fig. 5 also shows the magnitude of this function evaluated at the optimum value of $\xi$ used to generate the normalized noise reduction spectrum in Fig. 3. By comparing these two figures we see that noise reduction coincides with reduced correlation in the fluctuations. In fact, when the noise is maximally reduced at low frequency, the cross-spectral density approaches zero, indicating that the low-frequency fluctuations have been decorrelated by the transmission function. (Note: it is useful to take the log of the magnitude of (34), as in Fig. 5, because the large dynamic range at low frequencies is clearly seen, but an artifact of this is a sharp feature after the resonance where the function changes sign.) In addition, a new correlation at high frequency arises from the independent source of phase noise $\left[\tilde{\Delta}_{1}\right.$ term in $\left.(6 \mathrm{~b})\right]$ which is being converted to additive intensity noise by the transmission function.

The fact that the cross-spectral density is driven to zero at low frequencies when $\xi=\xi_{\text {opt }}$ can be understood by considering the fluctuations in the time domain. In the adiabatic limit, we have from $(4 a)-(4 c)$

$$
\begin{aligned}
& \hat{\rho}(t)=\Delta_{R} \\
& \dot{\varphi}(t)=\Delta_{I}+\alpha \Delta_{R}
\end{aligned}
$$

where $\hat{\rho}(t)=\tau_{R} \omega_{R}^{2} \rho(t)$ is a rescaled amplitude fluctuation in order that $\hat{\rho}(t)$ and $\dot{\varphi}(t)$ be dimensionally equivalent. Let $\sigma^{2}$ $=\left\langle\Delta_{R}^{2}\right\rangle=\left\langle\Delta_{l}^{2}\right\rangle$ be the variance of the Langevin terms (directly proportional to the integration bandwidth) so that

$$
\begin{aligned}
& \left\langle\hat{\rho}_{i}(t)^{2}\right\rangle=\sigma^{2} \\
& \left\langle\dot{\varphi}_{i}(t)^{2}\right\rangle=\sigma^{2}\left(1+\alpha^{2}\right)
\end{aligned}
$$

are the variances of the input field variables $\hat{\rho}_{i}(t)$ and $\dot{\varphi}_{i}(t)$. 
If the laser were operating in the tuned condition, corresponding to $\alpha=0$, then contours of constant probability density would be circular, as shown in Fig. 6 . For the detuned case, the contours are ellipses tilted with respect to the coordinate axes. The variance of the instantaneous frequency fluctuation is enhanced by $1+\alpha^{2}$ and the variance of the amplitude fluctuation is unchanged. This tilted ellipse represents the state of the input field, where the degree of tilt, and corresponding thinness of the ellipse measures the correlation between the fluctuations.

Using (23), (35a), and (35b), when $\xi=\xi_{\text {opt }}$, the output field variables are

$$
\begin{aligned}
& \hat{\rho}_{o}(t)=\frac{\Delta_{R}}{1+\alpha^{2}}-\frac{\alpha \Delta_{I}}{1+\alpha^{2}} \\
& \dot{\varphi}_{o}(t)=\Delta_{I}+\alpha \Delta_{R}
\end{aligned}
$$

which are clearly uncorrelated. The variances are given by

$$
\begin{aligned}
\left\langle\hat{\rho}_{o}(t)^{2}\right\rangle & =\frac{\sigma^{2}}{1+\alpha^{2}} \\
\left\langle\dot{\varphi}_{o}(t)^{2}\right\rangle & =\sigma^{2}\left(1+\alpha^{2}\right) .
\end{aligned}
$$

The output field ellipse is also shown in Fig. 6, For the optimum slope, we see that the transmission function has rotated the ellipse to align it with the coordinate axes. The output field variables $\hat{\rho}_{o}(t)$ and $\dot{\varphi}_{o}(t)$ are decorrelated while the variance of the amplitude fluctuation has simultaneously been reduced by the factor $1 /\left(1+\alpha^{2}\right)$.

\section{Effect of Power-Independent Linewidth}

To complete this discussion, we must consider the effect an extra, non-Schawlow-Townes, component of linewidth has on the RIN of the output field. We can foresee that, in general, the amount of noise reduction will diminish, since the extraneous linewidth is presumed to come from a phase noise source $\left[\tilde{\Delta}_{o}\right.$ term in (10)] which is not correlated with the other fluctuations. It is then straightforward to substitute this augmented instantaneous frequency fluctuation spectrum into the transformation equation (23) to compute the output RIN. In the low-frequency limit, we find analagous to (27)

$$
\operatorname{RIN}_{o}=\left[\left(\tau_{R} \omega_{R}^{2} \xi\right)^{2}(1+\beta)+\left(1+\alpha \tau_{R} \omega_{R}^{2} \xi\right)^{2}\right] \operatorname{RIN}_{i}
$$

where $\beta \equiv \Delta \omega_{o} / \Delta \omega_{\mathrm{ST}}$ is the ratio of the power-independent linewidth to the Schawlow-Townes linewidth. $\beta$ increases linearly with laser power, since $\Delta \omega_{\mathrm{ST}}$ has inverse power dependence. Evaluating the above relation at the slope which minimizes the output intensity noise,

$$
\xi_{\mathrm{opt}}=\frac{-1}{\tau_{R} \omega_{R}^{2}} \frac{\alpha}{1+\alpha^{2}+\beta}
$$

the maximum noise reduction is now given by

$$
\operatorname{RIN}_{o}=\frac{(1+\beta)}{\left(1+\alpha^{2}+\beta\right)} \operatorname{RIN}_{i}
$$

Thus, for nonzero $\beta$, the ability to reduce intensity noise is diminished compared with the ideal case (29). Furthermore, the amount of reduction is now a function of laser power, the net reduction becoming smaller as output power increases. This behavior will be shown to agree with measurements on a DFB laser diode in Section V.

For the complete output spectrum it can be easily seen that

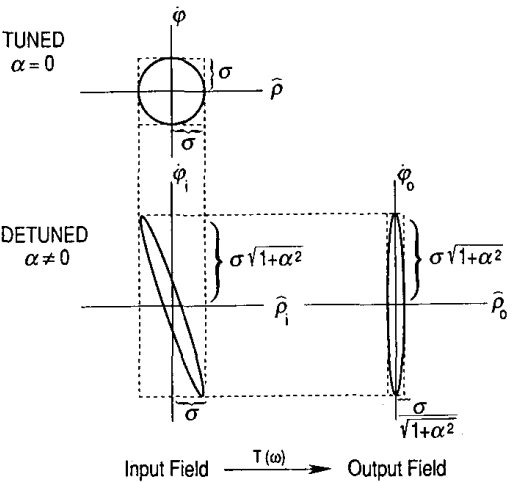

Fig. 6. Contours of constant probability density for tuned $(\alpha=0)$ and detuned $(\alpha \neq 0)$ operation. For detuned case, tilted ellipse of input field is rotated by $T(\omega)$ to decorrelate the variables and reduce variance of $\hat{\rho}_{o}(t)$ by $1 /\left(1+\alpha^{2}\right)$.

the presence of the extra linewidth component will add a uniform offset to the noise level by the amount $\xi^{2} \beta \Delta \omega_{\text {ST }}$ [see (24)]. Therefore, the magnitude of reduction at any frequency will decrease as the laser is run at higher bias.

To summarize the results of this section, we have studied the effect of an external, passive transmission function on the intensity noise of the laser field and found that large reductions below the intrinsic level are possible when the transmission function has the right slope. The method works by exploiting the inherent correlation between the field fluctuations. Optimum intensity noise reduction simultaneously decorrelates the amplitude and phase fluctuations at the output.

\section{INTENSITY NOISE REDUCTION BY INTRACAVITY LOSS ELEMENT}

A dispersive transmission function external to the laser cavity can reduce intensity noise, as was just shown. The same is true if the element is placed inside the cavity, where it then constitutes a dispersive loss. Contrary to the external case where the element acts on the field but does not affect the laser, the internal loss element influences the laser dynamics, and must be incorporated into the rate equations. The underlying mechanism for intensity noise reduction is similar in both cases, however, and the maximum amount of reduction is again given by the factor $1 /\left(1+\alpha^{2}\right)$.

This method for noise reduction will only be discussed briefly, because the effects of such an intracavity dispersive loss have been studied in work on "detuned loading" in semiconductor lasers [17], [18]. In fact, it was shown experimentally that phase noise reduction and modulation speed enhancement could be achieved simultaneously through such an approach [19]. The application to intensity noise reduction was not pursued in the literature. Here, our intent is to quantify how well the intensity noise is reduced when the loss element has the right slope. Intuitively, this mechanism for intensity noise reduction relies on amplitude-phase correlation, but contrary to the passive case there is a feedback mechanism through the optical gain. In other words, the enhanced phase noise (frequency jitter), which contains an image of the intensity noise, causes jitter in the instantaneous cavity loss owing to the dispersive loss function. The gain adjusts to compensate the fluctuating loss and so quiets the intensity noise, assuming the loss function has the right slope. 


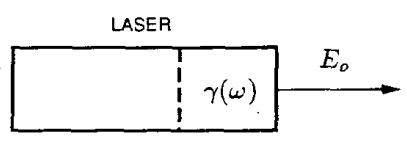

Fig. 7. Laser with frequency-dependent intracavity loss $\gamma(\omega)$. The intrinsic intensity noise can be reduced when $\gamma(\omega)$ has the right slope.

As mentioned above, there is reduction in the phase noise as well, but we will focus on the intensity noise here.

The dispersive intracavity loss $\gamma(\omega)$ is shown schematically in Fig. 7. The equation of motion for the slowly varying field amplitude (16) is given by [18]

$$
\dot{A}=\frac{1}{2}(G-\gamma(\omega))(1-i \alpha) A+\Delta
$$

where $\Delta=\Delta_{R}+i \Delta_{l}$ is the Langevin noise source. A linear expansion of $\gamma$ about the lasing frequency is assumed possible, so that

$$
\gamma(\omega)=\gamma\left(\omega_{L}\right)+2 \xi \delta \omega
$$

where $\xi$ is the slope of $\gamma$ and $\delta \omega$ is the complex instantaneous frequency deviation, given by

$$
\delta \omega=-i \frac{\dot{A}}{A}
$$

The small-signal equation of motion then results

$$
\dot{A}=\frac{G^{\prime} n(t)}{2} \frac{(1-i \alpha) A}{1-i \xi}+\frac{\Delta}{1-i \xi}
$$

where $G^{\prime}$ is the differential gain and $n(t)$ is the carrier density deviation. Substituting (16) for $A$ in terms of $\rho$ and $\varphi$, we find

$$
\dot{\rho}=\frac{G^{\prime} n(t)}{2} \frac{1+\alpha \xi}{1+\xi^{2}}+\frac{\Delta_{R}-\xi \Delta_{I}}{1+\xi^{2}} .
$$

At low frequencies where $n(t)$ and $\rho(t)$ track each other, this becomes [using (4c)]

$$
\rho(t)=\frac{1}{\tau_{R} \omega_{R}^{2}} \frac{\Delta_{R}-\xi \Delta_{I}}{1+\alpha \xi}
$$

where $\dot{\rho} \rightarrow 0$ in this adiabatic limit. The spectral density can then be found using the normalizations given in (5).

$$
W_{\rho \rho}=\frac{1}{\tau_{R}^{2} \omega_{R}^{4}} \frac{1+\xi^{2}}{(1+\alpha \xi)^{2}} \Delta \omega_{\mathrm{ST}} .
$$

Minimizing with respect to $\xi$ gives $\xi_{\text {opt }}=\alpha$ for the optimum slope, and so compared with the intrinsic RIN (spectral density when $\xi=0$ ), the optimum RIN is

$$
\operatorname{RIN}_{\mathrm{opt}}=\frac{1}{1+\alpha^{2}} \operatorname{RIN}_{\text {intrinsic }}
$$

which agrees with the theoretical minimum for the passive case (29). If the laser has an extraneous component of linewidth not due to spontaneous emission, then the remarks of Section III would apply here as well, and the maximum reduction would be diminished.

In summary, we have shown that an intracavity dispersive loss can potentially achieve the same level of intensity noise reduction as the passive transmission function studied in Section III. In the flat part of the noise spectrum, both methods predict that reduction by the factor $1 /\left(1+\alpha^{2}\right)$ is possible, assuming the laser does not possess a significant power-independent component of linewidth.

\section{EXPERIMENTAL RESULTS}

We have described two ways to reduce laser intensity noise below the intrinsic floor: an extracavity approach using a passive transmission element and an intracavity approach which incorporates a dispersive loss. In this section experimental results which verify the first approach are presented [1], [2]. The transmission function is implemented with a Michelson interferometer. Intensity noise from a DFB laser shows significant reduction which requires only a small optical path difference in the Michelson. The level of noise reduction as a function of frequency, laser bias, and transmission function slope agree with the theoretical predictions.

We note that Michelson interferometers are often used to characterize phase noise in semiconductor lasers [20], [21]. In these experiments, however, the interferometer is said to be strongly unbalanced, where the path difference is on the order of $10 \mathrm{~cm}$. In our experiment the interferometer is only slightly unbalanced. Optimum path differences for noise reduction are on the order of $1 \mathrm{~mm}$.

A schematic diagram of the experimental setup appears in Fig. 8. The single-mode laser source used in this experiment is an InGaAsP distributed feedback laser from Ortel operating at $1.3 \mu \mathrm{m}$. The threshold current is $21.8 \mathrm{~mA}$. The light is collimated by an antireflection coated lens and sent through an optical isolator (Newport ISO-13H) with $60 \mathrm{~dB}$ isolation to prevent feedback from influencing the measurement. After passing through a Michelson interferometer with a maximum $87 \%$ intensity transmission, the output light is focused onto a highquantum efficiency ( $90 \%$ ) InGaAs $\mathrm{p}-\mathrm{i}-\mathrm{n}$ detector. The noise photocurrent is amplified by a low-noise high-gain $(52 \mathrm{~dB}) \mathrm{am}$ plifier over the frequency band $0.01-1 \mathrm{GHz}$, and input to a microwave spectrum analyzer (HP 8558B). Lock-in detection is also employed to improve the sensitivity. With this arrangement, the intrinsic intensity noise from the DFB laser can be measured in both the excess and shot noise regimes, well above the thermal noise level of the detection system.

One arm of the Michelson is controlled by both a micrometer for coarse motion and a piezoelectric transducer for submicrometer motion. The dependence of intensity noise on the interferometer slope is then characterized by measuring the noise at a given frequency and bandwidth while scanning the Michelson by one wavelength starting from a known amount of path difference. If the scan begins at maximum transmission, for instance, then the input field samples a transmission slope ( $T_{R}^{\prime}$ in this case, not $\xi$ ), which changes smoothly from 0 to $m \delta / 2 c$ to 0 to $-m \delta / 2 c$ to 0 , where $\delta$ is the optical path difference, $c$ is the speed of light, and $m$ is the fringe visibility. In this way, a range of slopes can be tested in one scan, and the extremes of this range are set by the amount of path difference.

A linewidth versus power measurement was taken for the DFB laser which appears in Fig. 9. It shows that the linewidth varies inversely with power until it saturates at high power at a value of $7 \mathrm{MHz}$. This represents the power-independent linewidth component which will be shown to affect the magnitude of noise reduction that can be obtained.

Fig. 10 shows the results of an intensity noise measurement for a series of path differences at a laser bias of $23.3 \mathrm{~mA}$. The output power is $0.34 \mathrm{~mW}$ and the RIN is $-130 \mathrm{~dB} / \mathrm{Hz}$. The noise was measured in a $100 \mathrm{kHz}$ band at $130 \mathrm{MHz}$. Noise 


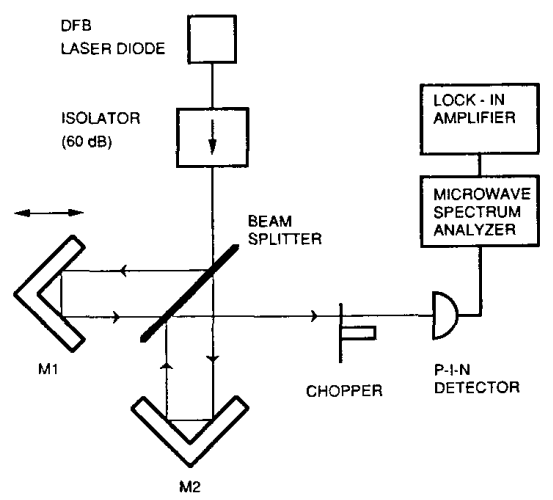

Fig. 8. Schematic diagram of the experimental arrangement including Michelson interferometer.

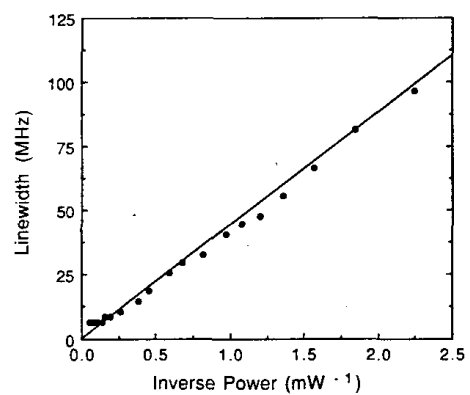

Fig. 9. Measured linewidth versus inverse power for DFB laser used in experiment. The high-power linewidth saturates at $7 \mathrm{MHz}$.

power is plotted as a function of transmitted intensity, proportional to the mean detector photocurrent. This is not the RIN, but the directly detected noise power which is related to RIN R $_{o}$ by multiplying the RHS of (39) by $|T|^{4}$. At zero path difference we see the intrinsic noise level as the light is merely attenuated by the Michelson. If the Michelson is unbalanced, then the dependence of noise level on transmission slope becomes apparent. Loops of noise are observed whereby the noise is reduced below the intrinsic level for positive slopes and enhanced when the slope is negative. At the extremes of transmitted intensity for a given loop, the noise level returns to the intrinsic value as it should since this corresponds to zero slope. As the path difference increases, the loops grow in area and the noise reduction switches to noise enhancement at some transmitted intensities.

With (39), theoretical noise loops can be calculated. Using $\alpha$ $=-2.3$, a value which is consistent with our data, $\tau_{R} \omega_{R}^{2}=34$ $\times 10^{9} \mathrm{rad} / \mathrm{s}\left(\omega_{R}\right.$ measured experimentally and $\tau_{R}$ taken from the literature [22]), and $\beta=0.35$ from the linewidth data, the calculated loops (Fig. 11) agree well with the measured loops.

In Fig. 12, cross sections of experimental and theoretical loops at the half-intensity transmission point are shown in terms of noise power normalized by the intrinsic noise level versus path difference. The optimum noise reduction is $7 \mathrm{~dB}$ at $4 \mathrm{~mm}$ path delay, which agrees with the theoretical calculation. Also shown are data from measurements at a higher bias of $25.7 \mathrm{~mA}$, where $\beta$ is $1.08, \tau_{R} \omega_{R}^{2}=82 \times 10^{9} \mathrm{rad} / \mathrm{s}$, output power $=0.83$ $\mathrm{mW}$, and RIN is $-140 \mathrm{~dB} / \mathrm{Hz}$. Because $\beta$ is bigger at higher bias, the amount of noise reduction decreases as predicted by (41). Also, the increase in $\tau_{R} \omega_{R}^{2}$ means the optimum slope is

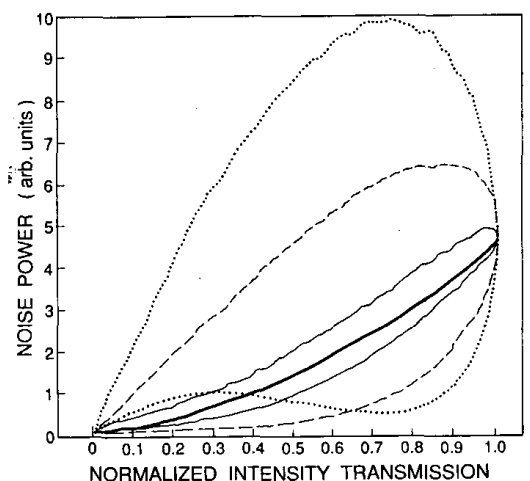

Fig. 10. Measured detector noise power versus normalized intensity transmission through the Michelson for optical path differences of $0 \mathrm{~mm}$ (intrinsic noise level), 1, 4, and $7 \mathrm{~mm}$. Loop areas increase with increasing path difference. Both reduction and enhancement of noise level are apparent.

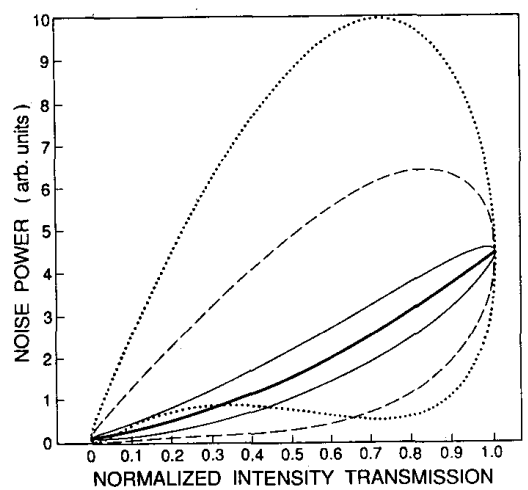

Fig. 11. Theoretical noise loops for optical path differences of $0,1,4$, and $7 \mathrm{~mm}$. Loop areas increase with increasing path difference.

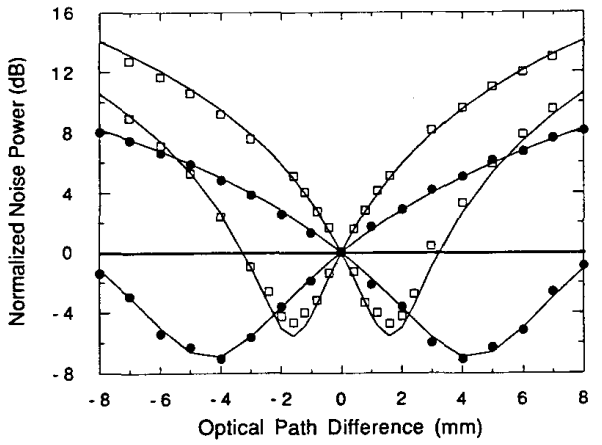

Fig. 12. Measured noise power at half-intensity transmission through Michelson versus optical path difference for laser bias of $23.3 \mathrm{~mA}$ (filled circles) and $25.7 \mathrm{~mA}$ (open squares). Power is normalized by its intrinsic value at zero path difference. Low-bias data show $7 \mathrm{~dB}$ noise reduction at $4 \mathrm{~mm}$ path difference. Theoretical calculations of noise power (lines) from (39) also appear.

decreased and occurs at a shorter path difference of $1.6 \mathrm{~mm}$, in accordance with (40). These data agree well with the theoretical plots. Note that the free-spectral range of the Michelson is 75 $\mathrm{GHz}$ for a path difference of $4 \mathrm{~mm}$. The linear expansion of the 




Fig. 13. Maximum noise power reduction versus frequency for $23.3 \mathrm{~mA}$ laser bias. Measured (dots) and theoretical (line) values are shown. Mag nitude of reduction decreases at higher frequencies in accordance with (24).

transmission function (14), on which the theoretical analysis of Section III is based, is therefore justified.

The amount of noise reduction continued to diminish as laser power increased. It can be seen however that for a laser having a smaller excess linewidth ( smaller $\beta$ at a given output power), larger amounts of noise reduction are possible at higher powers and at shorter path differences. These small path differences needed to achieve noise reduction suggest that a monolithically integrated version of this technique may be feasible.

For the low bias case at $23.3 \mathrm{~mA}$, Fig. 13 shows the maximum measured noise reduction as a function of frequency. The theoretical curve calculated from (24) and the known value of $\beta$ also appears. As predicted, the amount of reduction diminishes at higher frequencies where the intrinsic noise spectrum is no longer flat. Note that an additional benefit from a laser with a smaller component of excess linewidth is that noise reduction becomes possible at high bias levels. The resonance frequency is therefore pushed out to higher frequencies, leading to noise reduction over a wider bandwidth.

Using a Michelson interferometer, we have reduced intensity noise from a DFB laser by as much as $7 \mathrm{~dB}$, which agrees with the theoretical results of Section III. The measured dependence of the noise level on interferometer slope, laser bias, and frequency are also accounted for by theory. A component of powerindependent linewidth, $7 \mathrm{MHz}$ for this DFB laser, inhibits intensity noise reduction at higher bias levels. A laser with a smaller extraneous linewidth would show better reduction at higher bias.

\section{Conclusion}

In conclusion, we have discussed a simple technique, amplitude-phase decorrelation, for reducing the intensity noise floor in a free-running semiconductor laser. Taking advantage of the inherent coupling between the amplitude and phase fluctuations in the laser field, the intensity noise can potentially be reduced by the factor $1 /\left(1+\alpha^{2}\right)$, independent of laser power, by using a passive external element with a frequency-dependent transmission. Optimum intensity noise reduction results in decorrelation of the fluctuations. This technique was shown to share some conceptual similarities with detuned loading, where a dispersive loss function is placed inside the laser cavity. In that case the maximum intensity noise reduction is also $1 /\left(1+\alpha^{2}\right)$. In practice, the presence of a power-independent linewidth will limit the amount of reduction that can be achieved, and the magnitude of reduction will diminish as laser power increases. However, we verify that the intensity noise from a DFB laser diode at low bias can be reduced by as much as $7 \mathrm{~dB}$, in agreement with theory, when the passive technique is implemented with a Michelson interferometer. A semiconductor laser with a smaller component of extraneous linewidth should show even more intensity noise reduction over a wider range of output powers.

Note Added in Proof: The authors performed amplitude-phase decorrelation experiments on a DFB laser with a larger $\alpha$ parameter than the device discussed here, which showed up to a $10.8 \mathrm{~dB}$ reduction in the intensity noise level.

\section{ACKNOWLEDGMENT}

The authors thank H. Blauvelt and J. Paslaski of Ortel Corporation for DFB lasers and mounting the detectors used in the experiment, and J. Dawson for technical assistance.

\section{REFERENCES}

[1] K. J. Vahala and M. A. Newkirk, "Intensity noise reduction in semiconductor lasers by amplitude-phase decorrelation," presented at XVII Int. Quantum Electron. Conf., May 21-25, 1990, Anaheim, CA, postdeadline paper QPDP29.

[2] _- "Intensity noise reduction in semiconductor lasers by amplitude-phase decorrelation," Appl. Phys. Lett., vol. 57, pp. 974976, 1990

[3] D. Welford and A. Mooradian, "Output power and temperature dependence of the linewidth of single frequency $\mathrm{cw}(\mathrm{GaAl}) \mathrm{As}$ diode lasers," Appl. Phys. Lett., vol. 40, pp. 865-867, 1982.

[4] C. Harder, K. Vahala, and A. Yariv, "Measurement of the linewidth enhancement factor $\alpha$ of semiconductor lasers," Appl. Phys. Lett., vol. 42, pp. 328-330, 1983.

[5] T. L. Koch and J. E. Bowers, "Nature of wavelength chirping in directly modulated semiconductor lasers," Electron. Lett., vol. 20, pp. 1038-1040, 1984.

[6] C. H. Henry, "Theory of the linewidth of semiconductor lasers," IEEE J. Quantum Electron., vol. QE-18, pp. 259-264. 1982 .

[7] M. Osiński and J. Buus, "Linewidth broadening factor in semiconductor lasers-An overview," IEEE J. Quantum Electron., vol. QE-23, pp. 9-29, 1987.

[8] K. Vahala and A. Yariv, "Semiclassical theory of noise in semiconductor lasers-Part I," IEEE J. Quantum Electron., vol. QE-19, pp. 1096-1101, 1983.

[9] _ - "Semiclassical theory of noise in semiconductor lasersPart II,' IEEE J. Quantum Electron., vol. QE-19, pp. 1102$1109,1983$.

[10] C. H. Henry, "Theory of the phase noise and power spectrum of a single mode injection laser," IEEE J. Quantum Electron., vol. QE-19, pp. 1391-1397, 1983.

[11] D. Welford and A. Mooradian, "Observation of linewidth broadening in $(\mathrm{GaAl})$ As diode lasers due to electron number fluctuations," Appl. Phys. Lett., vol. 40, pp. 560-562, 1982.

[12] K. Vahala and A. Yariv, "Occupation fluctuation noise: A fundamental source of linewidth broadening in semiconductor lasers," Appl. Phys. Lett., vol. 43, pp. 140-142, 1983.

[13] K. Uomi, S. Sasaki, T. Tsuchiya, M. Okai, M. Aoki, and N. Chinone, "Spectral linewidth reduction by low spatial hole burning in $1.5 \mu \mathrm{m}$ multi-quantum-well $\lambda / 4$-shifted DFB lasers," Electron. Lett, , vol. 26, pp. 52-53, 1990.

[14] S. Machida and Y. Yamamoto, "Observation of amplitude squeezing in a constant-current-driven semiconductor laser," Phys. Rev. Lett., vol. 58, pp. 1000-1003, 1987.

[15] K. Vahala and M. Newkirk, unpublished

[16] K. Kikuchi and T. Okoshi, "Measurement of FM noise, AM noise, and field spectra of $1.3 \mu \mathrm{m}$ InGaAsP DFB lasers and determination of the linewidth enhancement factor," IEEE J. Quantum Electron., vol. QE-21, pp. 1814-1819, 1985.

[17] K. Vahala and A. Yariv, "Detuned loading in coupled cavity semiconductor lasers-effect on quantum noise and dynamics," Appl. Phys. Lett., vol. 45, pp. 501-503, 1984. 
[18] K. Vahala, "Dynamic and spectral features of semiconductor lasers," Ph.D. dissertation, California Instit. Technol., Pasadena, CA, 1985.

[19] K. Vahala, J. Paslaski, and A. Yariv, "Observation of modulation speed enhancement, frequency modulation suppression, and phase noise reduction by detuned loading in a coupled-cavity semiconductor laser," Appl. Phys. Lett., vol. 46, pp. 1025-1027. 1985.

[20] B. Daino, P. Spano, M. Tamburrini, and S. Piazzolla, "Phase noise and spectral line shape in semiconductor lasers,"' IEEE $J$. Quantum Electron., vol. QE-19, pp. 266-270, 1983.

[21] P. Spano, S. Piazzolla, and M. Tamburrini, "Phase noise in semiconductor lasers: A theoretical approach," IEEE J. Quantum Electron., vol. QE-19, pp. 1195-1199, 1983.

[22] R. J. Lang, H. P. Mayer, H. Schweizer, and A. P. Moser, "Measurement of the relaxation resonance, damping, and nonlinear gain coefficient from the sidebands in the field spectrum of a $1.3 \mu \mathrm{m}$ InGaAsP distributed feedback laser," Appl. Phys. Lett., vol. 54 pp. 1845-1847, 1989.

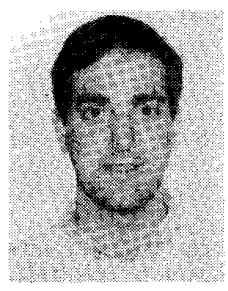

Michael A. Newkirk was born in Palo Alto, $\mathrm{CA}$. He received the $\mathrm{B}$. A. degree in physics from Williams College, Williamstown, MA, in 1983.

At the Fundamental Research Laboratory, GTE Laboratories, Waltham, MA (19841986), he was involved in the study of semiconductor lasers and the electronic and nonlinear optical properties of polymeric crystals. Currently, he is a Ph.D. candidate in applied physics at the California Institute of Technol- ogy, Pasadena. His research centers on semiconductor laser modulation dynamics and field fluctuations.

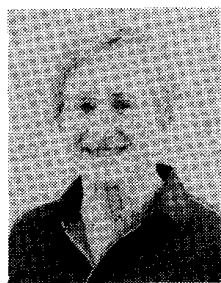

Kerry J. Vahala (S'82-M'84-S'84-M'85) received the B.S., M.S., and Ph.D. degrees in 1980,1981 , and 1985 , respectively, all in physics, from the California Institute of Technology, Pasadena.

He is now an Associate Professor with the Department of Applied Physics, California Institute of Technology. His research interests include the dynamic and spectral properties of semiconductor lasers and the physics of lower dimensional solid-state electronic systems including quantum-well, wire, and dot structures.

Dr. Vahala is the recipient of the Presidential Young Investigator Award, the Office of Naval Research Young Investigator Award, two Teaching Awards from the Associated Students of the California Institute of Technology, and is the first recipient of the Richard P. Feynman-Hughes Fellowship. 